\title{
VANISHING THEOREMS, A THEOREM OF SEVERI, AND THE EQUATIONS DEFINING PROJECTIVE VARIETIES
}

\author{
AARON BERTRAM, LAWRENCE EIN, AND ROBERT LAZARSFELD
}

\section{CONTENTS}

Introduction

0 . Notation and conventions

1. A variant of Severi's theorem

2. Applications to subvarieties of projective space

3. Normal generation of adjoint bundles

References

\section{INTRODUCTION}

In 1903 Severi [S] proved that if $X, X^{\prime} \subset \mathbb{P}^{r}$ are smooth surfaces whose union is the complete intersection of $r-2$ hypersurfaces of degrees $d_{1}, \ldots, d_{r-2}$, then hypersurfaces of degree $k \geq \sum d_{i}-r$ cut out a complete linear series on $X$ (cf. [SR, XIII.9.8]). The purpose of this paper is first of all to show that elementary arguments using the Kodaira vanishing theorem lead to a simple variant of Severi's statement (Theorem 7 below) which extends it in serval directions. More importantly, we hope to convince the reader that this result has a surprising number of applications to questions involving the equations defining projective varieties.

Consider to begin with a smooth complex projective variety $X \subset \mathbb{P}^{r}$ of dimension $n$ and codimension $e=r-n$. In this setting, our theorem asserts the vanishing of the higher cohomology of suitable twists of the ath power of the ideal sheaf $\mathscr{I}_{X}$ of $X$ in $\mathbb{P}^{r}$.

Proposition 1. Assume that $X$ is cut out scheme-theoretically in $\mathbb{P}^{r}$ by hypersurfaces of degrees $d_{1} \geq d_{2} \geq \cdots \geq d_{m}$. Then

$$
H^{i}\left(\mathbb{P}^{r}, \mathcal{F}_{X}^{a}(k)\right)=0 \quad \text { for } i \geq 1 \text { provided } k \geq a d_{1}+d_{2}+\cdots+d_{e}-r .
$$

Note that only the degrees of the first $e=\operatorname{codim}\left(X, \mathbb{P}^{r}\right)$ defining equations come into play here. When $n=2$ and $a=1$ this is a consequence of Severi's

Received by the editors November 29, 1990.

1991 Mathematics Subject Classification. Primary 14F17.

The authors were, respectively, supported by an N.S.F. Postdoctoral Fellowship, a Sloan Fellowship and N.S.F. Grant DMS 89-04243, and N.S.F. Grant DMS 89-02551. 
result. We remark that if $d_{1}=\cdots=d_{e}=d$-a case that suffices for many of the applications-then there is a particularly quick proof of the proposition (see Remark 1.10).

Proposition 1 has a number of corollaries. First of all, taking $a=1$, we get

Corollary 2. Keeping notation as above, if $d_{1}+\cdots+d_{e} \leq r+1$, then $X$ is projectively normal. If $d_{1}+\cdots+d_{e} \leq r$, then $X$ is projectively Cohen-Macaulay.

For example, if $X^{n} \subset \mathbb{P}^{2 n+1}$ is cut out by quadrics, then $X$ is projectively normal, a fact illustrated by numerous classical examples.

Corollary 2 in turn leads to a simplification and strengthening of the "Babylonian tower" theorems of Hartshorne and Barth-Van de Ven [B] concerning subvarieties of very low degree in projective space. Specifically, suppose that $X \subset \mathbb{P}^{r}$ as above has degree $d$. Then, as is well known, $X$ is defined by hypersurfaces of degree $d$, and hence it follows from the corollary that $X$ is projectively Cohen-Macaulay as soon as $d e \leq r$. Elementary estimates for Cohen-Macaulay rings then show that the homogeneous ideal $I_{X}$ of $X$ has $\leq d e$ minimal generators. On the other hand, an (elementary) theorem of Faltings [F] states that if $X \subset \mathbb{P}^{r}$ is defined scheme-theoretically by $r / 2$ or fewer equations, then $X$ is a complete intersection. Hence

Corollary 3. Assume that $X \subset \mathbb{P}^{r}$ is a smooth variety of degree $d$, dimension $n$, and codimension $e$. If

$$
d \leq \frac{r}{2 e} \quad\left[=\frac{n}{2 e}+\frac{1}{2}\right],
$$

then $X$ is a complete intersection.

Hartshorne (cf. [H]) showed that if one fixes $d$ and $e$ and lets $n$ go to infinity, then eventually $X$ becomes a complete intersection. Barth and Van de Ven [B] obtained the same conclusion under the explicit assumption that $d(d-1)<2 n / 5$. When $e=2$, Ran [R1] proved a much stronger inequality, which was strengthened in [HS]. The motivation of these results is of course the (still wide open) conjecture that any smooth subvariety of sufficiently small codimension in projective space is a complete intersection. The possiblity of using Faltings's theorem in the present context was suggested to us by F. L. Zak (compare [F12]). Flenner [Fl1, Fl2] and Sato [Sat] have established Babylonian tower theorems in considerably more general settings, for example weighted projective spaces. It would be interesting to find analogous extensions of Proposition 1.

The next applications concern the Castelnuovo-Mumford regularity of $X \subset$ $\mathbb{P}^{r}$. Recall that one says that $X$ is $k$-regular if $H^{i}\left(\mathbb{P}^{r}, \mathscr{J}_{X}(k-i)\right)=0$ for $i>0$. The significance of this concept stems from the fact that the regularity of $X$ governs the complexity of computing the syzygies and other invariants of $X$ (cf. [BS1, BS2]). For instance, a theorem of Mumford's [M] states that $X$ is $k$-regular if and only if for every $p \geq 0$ the minimal generators of the $p$ th module of syzygies of the homogeneous ideal $I_{X}$ occur in degrees $\leq k+p$. It is 
therefore of interest to bound the regularity of $X$ in terms of the degrees of its defining equations. For an arbitrary scheme, this regularity can be horrendously large; there are examples due to Mayr-Meyer-Bayer-Stillman [BS1] of schemes $X \subset \mathbb{P}^{r}$ defined by hypersurfaces of degree $d$ with regularity $\geq(d-2)^{2^{(r / 10)}}$. However, experience with actual computations suggests that for the sorts of varieties that occur in natural problems-e.g. smooth varieties-the regularity grows much more slowly with $d$ and $r$. This empirical observation is justified by again taking $a=1$ in Proposition 1, which yields an optimal bound when $X$ is smooth.

Corollary 4. Assume as above that $X \subset \mathbb{P}^{r}$ is a smooth variety of dimension $n$ and codimension $e$ defined by hypersurfaces of degrees $d_{1} \geq d_{2} \geq \cdots \geq d_{m}$. Then

(i) $X$ is $\left(d_{1}+\cdots+d_{e}-e+1\right)$-regular; and

(ii) $X$ fails to be $\left(d_{1}+\cdots+d_{e}-e\right)$-regular if and only if $X$ is the complete intersection of hypersurfaces of degrees $d_{1}, d_{2}, \ldots, d_{e}$.

In other words, we may say that complete intersections have the "worst" regularity among all smooth varieties defined by equations of given degrees. Previous work on the regularity of $X$ has centered around estimates involving the degree of $X$ (cf. [GLP, P, L1, and BM]). Here Proposition 1 implies a slight strengthening (Corollary 2.1) of a bound of Mumford's, which however is presumably not optimal.

An application of a rather different flavor concerns the Hodge type of $X \subset \mathbb{P}^{r}$. Let $U=\mathbb{P}^{r}-X$ denote the complement of $X$. One says that $X$ has Hodge type $\operatorname{Ht}(X) \geq t$ if $F^{t} H_{c}^{i}(U)=H_{c}^{i}(U) \quad \forall i \geq 0$, where $F$ is the Hodge-Deligne decreasing filtration. Motivated by some arithmetic results, Deligne and Dimca [DD] conjecture that if $X \subset \mathbb{P}^{r}$ is an arbitrary algebraic set defined by equations of degrees $d_{1} \geq d_{2} \geq \cdots \geq d_{m}$, then

$$
\mathrm{Ht}(X) \geq\left[\left(r+1-\sum d_{i}\right) / d_{1}\right] .
$$

They prove this when $X$ is a hypersurface, and Esnault $[E]$ has established the conjecture when $X$ is a complete intersection. In the special case when $X$ is smooth, Esnault's results combined with Proposition 1 yield

Corollary 5. If $X \subset \mathbb{P}^{r}$ is a smooth connected variety of codimension $e$ defined scheme-theoretically by hypersurfaces of degrees $d_{1} \geq d_{2} \geq \cdots \geq d_{m}$, then $\mathrm{Ht}(X) \geq\left[\left(r+1-\sum_{i=1}^{e} d_{i}\right) / d_{1}\right]$.

This inequality implies $(*)$, but of course the hypotheses on $X$ are much stronger than what one would like.

Changing gears somewhat, we consider finally the normal generation of adjoint bundles on a smooth projective variety $X$ of dimension $n$. There has been considerable interest recently in the projective normality and defining equations of algebraic curves and other varieties (cf. [M, G, L2, KMF, Btl]). The prototypical result here is an old theorem of Castelnuovo et al. to the effect that a 
line bundle of degree $\geq 2 g+1$ on a smooth curve of genus $g$ defines a projectively normal embedding. It is natural to ask for analogous statements in higher dimensions. Mukai observed that the known theorems deal with embeddings defined by bundles of the type $K_{X} \otimes P$, where $P$ is an explicit multiple of a suitably positive bundle. He suggested that one should aim for general results having this shape. In this direction, we prove that if $A$ is a very ample line bundle on $X$, then $K_{X} \otimes A^{\otimes k}$ is normally generated as soon as $k \geq n+1$.

Proposition 6. Let $A$ be a very ample line bundle on $X$, and let $B$ and $C$ be arbitrary numerically effective line bundles on $X$. Then the natural multiplication map

$H^{0}\left(X, K_{X} \otimes A^{\otimes k} \otimes B\right) \otimes H^{0}\left(X, K_{X} \otimes A^{\otimes m} \otimes C\right) \rightarrow H^{0}\left(X, K_{X}^{\otimes 2} \otimes A^{\otimes k+m} \otimes B \otimes C\right)$ is surjective provided that $k, m \geq n+1$. In particular, if $k \geq n+1$, then $K_{X} \otimes A^{\otimes k} \otimes B$ defines a projectively normal embedding of $X$ provided that it is very ample, i.e., if $(X, A, B) \neq\left(\mathbb{P}^{n}, \mathscr{O}_{\mathbb{P}^{n}}(1), \mathscr{O}_{\mathbb{P}^{n}}\right)$.

We show that the same statement holds with one exception when $k, m \geq n$. These results have been obtained independently by Andreatta, Ballico, and Sommese [AS, ABS]. Analogous assertions for defining equations for higher syzygies are established by different methods in [EL], to which we refer for a fuller discussion of the background and earlier work on these questions.

The propositions are consequences of

Theorem 7. Let $M$ be a smooth complex projective variety, let $A$ be an ample line bundle on $M$, and let $L$ be a globally generated line bundle on $M$. Consider a smooth subvariety $X \subset M$ of codimension $e$, with ideal sheaf $\mathscr{I}_{X}=\mathscr{I}_{X / M}$. Suppose that $X$ is defined scheme-theoretically in $M$ by the vanishing of $m$ sections $s_{i} \in H^{0}\left(M, L^{\otimes d_{i}}\right)$, where $d_{1} \geq d_{2} \geq \cdots \geq d_{m}$. Then

$$
H^{i}\left(M, \mathcal{F}_{X}^{a} \otimes K_{M} \otimes L^{\otimes k} \otimes A\right)=0 \quad \text { for } i \geq 1
$$

provided $k \geq a d_{1}+d_{2}+\cdots+d_{e}$.

In fact, it is enough here that $A$ be big and nef. Proposition 1 follows immediately. For Proposition 6, one applies the theorem to the diagonal $\Delta \subset X \times X$, which is cut out by sections of $L=\left(p_{1}\right)^{*} A \otimes\left(p_{2}\right)^{*} A$ as long as $A$ is very ample. In this setting, the analogous statements involving higher powers of $\mathscr{I}_{\Delta}$ yield information about the surjectivity of the Wahl map and its generalizations (see Corollary 3.4).

As for Theorem 7, the idea is to consider the blowing-up $p: P=\mathrm{Bl}_{X}(M) \rightarrow$ $M$ of $M$ along $X$, and to apply the Kodaira-Ramanujan-Kawamata-Viehweg vanishing theorem. When $d_{1}=d_{2}=\cdots=d_{m}=d$, one can apply vanishing directly on $P$, in which case the result is practically immediate. In general, as in Severi's set-up, we work on the blowing-up $Y \subset P$ of the variety residual to $X$ in the complete intersection of suitable sections $t_{i} \in H^{0}\left(M, \mathscr{J}_{X} \otimes L^{\otimes d_{i}}\right)$. We remark that a similar argument yields a direct generalization (Proposition 
1.11) of Severi's theorem, as well as a generalization (Proposition 1.12) of the Griffiths vanishing theorem [Griff] for globally generated vector bundles.

The possiblity of applying vanishing theorems on a blow-up to study the equations vanishing on a subvariety is evidently not a new idea. For example, much more sophisticated arguments along these lines occur in the work of Esnault and Viehweg [EV1, EV2] on Dyson's lemma. They and others (including some of the present authors) have been at least implicitly aware for some time of the special case $d_{1}=d_{2}=\cdots=d_{m}=d$ of Theorem 7. However, the applicability of these ideas to the sort of projective-geometric questions considered above seems not to have recognized, and it is here that we post our main claim to novelty.

The Severi-type Theorem 7 and some variants are proven in $\S 1$. Section 2 contains the applications to subvarieties of projective space, viz. Proposition 1 and Corollaries 2, 3, 4, and 5. We prove the normal generation of adjoint bundles (Proposition 6) and some related results in $\S 3$.

We wish to thank D. Bayer, D. Butler, D. Eisenbud, H. Pinkham, F. L. Zak, and especially $\mathrm{H}$. Esnault for valuable discussions and encouragement.

\section{Notation AND CONVENTIONS}

(0.1) We work throughout over the complex numbers.

(0.2) Let $X$ be a projective variety of dimension $n$. If $L$ is a line bundle on $X$, we write $L^{k}$ for the $k$-fold tensor power $L^{\otimes k}$ of $L$. If $k \geq 0$, then as usual $L^{-k}=\left(L^{*}\right)^{k}$. Recall that $L$ is nef if $c_{1}(L) \cdot \Gamma \geq 0$ for every irreducible curve $\Gamma \subset X . L$ is big if for some $m>0$ the rational map $\Phi_{|m L|}: X \rightarrow \mathbb{P} H^{0}\left(L^{m}\right)$ is birational. If $L$ is nef, this is equivalent to asking that $\int c_{1}(L)^{n}>0$. (See [Mori, (1.9)] for a fuller discussion.)

(0.3) When $X$ is smooth, we denote by $K_{X}$ the canonical bundle on $X$. We will frequently use the Kawamata-Viehweg vanishing theorem $[\mathrm{K}, \mathrm{V}]$, which states that if $L$ is a big and nef line bundle on a smooth projective variety, then $H^{i}\left(X, K_{X} \otimes L\right)=0$ for $i \geq 1$.

(0.4) Let $L_{1}, \ldots, L_{m}$ be line bundles on a variety $M$. We say that a subvariety $X \subset M$ with ideal sheaf $\mathscr{I}_{X}$ is scheme-theoretically cut out by sections $s_{i} \in H^{0}\left(M, L_{i}\right)$ if the $s_{i}$ vanish on $X$, and if the sheaf homomorphism $\bigoplus L_{i}^{*} \rightarrow \mathscr{I}_{X}$ determined by the $s_{i}$ is surjective.

\section{A VARIANT OF SERVERI'S THEOREM}

In this section we work in the setting of Theorem 7 from the Introduction. Thus, $M$ is a smooth complex projective variety of dimension $r, A$ is a big and nef line bundle on $M$, and $L$ is a globally generated line bundle on $M$. We consider a smooth subvariety $X \subset M$ of pure dimension $n$ and codimension 
$e=n-r$, and we suppose given the surjective map

$$
\bigoplus_{i=1}^{m} L^{-d_{i}} \rightarrow \mathscr{I}_{X} \rightarrow 0
$$

where $d_{1} \geq d_{2} \geq \cdots \geq d_{m}>0$. Our purpose now is to prove

Theorem 1.2. Under the hypotheses just stated $H^{i}\left(M, \mathscr{J}_{X}^{a} \otimes K_{M} \otimes L^{k} \otimes A\right)=0$ for $a, i \geq 1$ and $k \geq a d_{1}+d_{2}+\cdots+d_{e}$.

As in the introduction, $\mathscr{F}_{X}^{a}$ denotes the ath power of the ideal sheaf $\mathscr{F}_{X}$.

We start by recording two elementary lemmas.

Lemma 1.3. Let $P$ be a smooth variety, let $A$ be a big and nef line bundle on $P$, and let $\vartheta$ be a base-point free linear system of divisors on $P$. Then the restriction $\mathrm{A} / \mathrm{D}$ of $A$ to a general element $D \in \vartheta$ is again big and nef.

Proof. This is clear given the fact (cf. [Mori, (1.9), (1.9.1)]) that a nef line bundle $A$ is big if and only if there exists a natural number $d>0$ and a very ample divisor $H$ such that $A^{d}=H \otimes N$, where $h^{0}(N) \neq 0$.

Lemma 1.4. Let $X \subset M$ be a smooth codimension $e$ subvariety of a smooth variety $M$, let $f: P=\mathrm{Bl}_{X}(M) \rightarrow M$ be the blowing-up of $M$ along $X$, and let $E \subset P$ be the exceptional divisor. If $0 \leq t \leq e-1$, then

$$
H^{i}\left(P, f^{*} F \otimes \mathscr{O}_{p}(t E)\right)=H^{i}(M, F) \quad \forall i
$$

for any locally free sheaf $F$ on $M$.

Proof. By the projection formula and the Leray spectral sequence, it is enough to show that $f_{*} \mathscr{O}_{P}(t E)=\mathscr{O}_{M}$ and $R^{i} f_{*} \mathscr{O}_{P}(t E)=0$ for $i>0$ and $t$ in the indicated range. When $t=0$ that is well known. For $t \geq 1$ one argues by induction from the sequence

$$
0 \rightarrow \mathscr{O}_{P}((t-1) E) \rightarrow \mathscr{O}_{P}(t E) \rightarrow \mathscr{O}_{E}(t E) \rightarrow 0
$$

using the facts that $E=\mathbb{P} N$ is the projectivization of a rank $e$ vector bundle $N$ on $X$, and that $\mathscr{O}_{E}(E)=\mathscr{O}_{\mathbb{P} N}(-1)$.

Now we turn to the

Proof of Theorem 1.2. Let $f: P=\mathrm{Bl}_{X}(M) \rightarrow M$ be the blowing-up of $M$ along $X$, and denote by $E \subset P$ the exceptional divisor. Recalling again that $f_{*} \mathscr{O}_{P}=\mathscr{O}_{M}$ and $R^{i} f_{*} \mathscr{O}_{P}=0$ for $i>0$ because $X$ is smooth, one finds by induction on $a$ the well-known fact that if $a>0$ then $f_{*} \mathscr{O}_{P}(-a E)=\mathscr{F}_{X}^{a}$ and $R^{i} f_{*} \mathscr{O}_{P}(-a E)=0$ for $i>0$. The projection formula and the Leray spectral sequence therefore yield

$$
H^{*}\left(P, f^{*} B(-a E)\right)=H^{*}\left(M, \mathcal{I}_{X}^{a} \otimes B\right)
$$

for any line bundle $B$ on $M$. With this in mind, the issue is to prove that $H^{i}\left(P, f^{*}\left(K_{M} \otimes L^{\otimes k} \otimes A\right)(-a E)\right)=0$ for $i \geq 1$ and $k \geq a d_{1}+d_{2}+\cdots+d_{e}$. 
To this end, let $B_{i}=f^{*} L^{d_{i}}(-E)$. Then

$$
B_{i} \otimes\left(B_{j}\right)^{*}=f^{*}\left(L^{d_{i}-d_{j}}\right) \quad \text { is base-point free for } i \geq j
$$

thanks to the facts that $L$ is base-point free and $d_{i} \geq d_{j}$ when $i \geq j$. On the other hand, the sections $s_{i} \in H^{0}\left(M, L^{d_{i}}\right)$ defining $X$ give arise in the natural way to effective divisors $F_{i} \in\left|B_{i}\right|$, and the existence of the surjective map (1.1) means precisely that

$$
F_{1} \cap F_{2} \cap \cdots \cap F_{m}=\varnothing .
$$

We now proceed in several steps.

Claim 1.5. Let $D_{i} \in\left|B_{i}\right|$ be a general divisor, and for $1 \leq s \leq e$, set

$$
Y_{s}=D_{1} \cap D_{2} \cap \cdots \cap D_{s} \text {. }
$$

Then by choosing the $D_{i}$ suitably, we may assume that

(i) $Y_{s}$ is smooth (but possibly disconnected) of pure codimension $s$ in $P$;

(ii) for any irreducible component $Y \subset Y_{s}$, the restriction $f^{*} A \mid Y$ is big and nef;

(iii) no component of $Y_{s}$ is contained in the exceptional divisor $E$; and

(iv) $Y_{s} \cap F_{s+1} \cap \cdots \cap F_{m}=\varnothing$.

We prove Claim 1.5 by induction on $s$. It follows to begin with from $(*)$ and $(* *)$ that $B_{1}$ is globally generated, and hence (i), (iii), and (iv) are clear when $s=1$. Statement (iii) follows in the case $s=1$ from (1.3) and the fact that $f^{*} A$ is big and nef. Assume inductively that Claim 1.5 is known for a given $s$. Denoting by $\operatorname{Bs}\left(B_{i}\right)$ the base-locus of $B_{i}$, it is a consequence of $(*)$ that $\operatorname{Bs}\left(B_{s+1}\right) \subset F_{s+1} \cap \cdots \cap F_{m}$. Therefore, thanks to (iv), $B_{s+1} \mid Y_{s}$ is base-point free and, in fact, $B_{s+1} \mid Y_{s}$ is generated by $H^{0}\left(P, B_{s+1}\right)$. But then by taking $D_{s+1} \in\left|B_{s+1}\right|$ to be sufficiently general, we can certainly arrange that $Y_{s+1}=Y_{s} \cap D_{s+1}$ satisfies (i)-(iv).

Now fix $Y=Y_{e}=D_{1} \cap D_{2} \cap \cdots \cap D_{e}$ satisfying the assertions of Claim 1.5, and the set $d=d_{1}+d_{2}+\cdots+d_{e}$.

Claim 1.6. If $k \geq a d_{1}+d_{2}+\cdots+d_{e}$, then

$$
H^{i}\left(P, f^{*}\left(K_{M} \otimes L^{k} \otimes A\right) \otimes \mathscr{O}_{Y}(-a E)\right)=0 \text { for } a, i \geq 1 .
$$

We prove this by applying the Kawamata-Viehweg vanishing theorem on (each connected component of) $Y$. To this end, use adjunction and the formula for the canonical divisor of a blow-up along a smooth center to compute

$$
\begin{aligned}
K_{Y} & =K_{P} \otimes B_{1} \otimes \cdots \otimes B_{e} \mid Y \\
& =f^{*} K_{M} \otimes \mathscr{O}_{P}((e-1) E) \otimes\left(f^{*} L^{d} \otimes \mathscr{O}_{P}(-e E)\right) \mid Y \\
& =F^{*}\left(K_{M} \otimes L^{d}\right) \otimes \mathscr{O}_{P}(-E) \mid Y .
\end{aligned}
$$

(If $Y$ is disconnected, the restriction of the bundle appearing on the right-hand side computes the canonical bundle of any component of $Y$.) Therefore $f^{*}\left(K_{M} \otimes L^{k} \otimes A\right) \otimes \mathscr{O}_{P}(-a E) \otimes \mathscr{O}_{Y}=K_{Y} \otimes f^{*}\left(A \otimes L^{k-a d_{1}-d_{2}-\cdots-d_{e}}\right) \otimes\left(B_{1}\right)^{a-1}$. 
Recalling that $B_{1}=f^{*} L^{d_{1}}(-E)$ is base-point free and that $f^{*} A \mid Y$ is big and nef, it follows that if $k \geq a d_{1}+d_{2}+\cdots+d_{e}$. Then $f^{*}\left(K_{M} \otimes L^{k} \otimes A\right) \otimes$ $\mathscr{O}_{P}(-a E) \otimes \mathscr{O}_{Y}$ is of the form $K_{Y} \otimes C$, where $C$ is nef and big. Hence Claim 1.6 follows from [K] or [V].

Consider next the exact sequence $0 \rightarrow \mathscr{I}_{Y} \rightarrow \mathscr{O}_{P} \rightarrow \mathscr{O}_{Y} \rightarrow 0$. Twisting through by $f^{*}\left(K_{M} \otimes L^{k} \otimes A\right)(-a E)$, the theorem will follow from Claim 1.6 as soon as we establish the vanishing

$$
\begin{aligned}
& \text { If } a \geq 1 \text { and } k \geq a d_{1}+d_{2}+\cdots+d_{e}, \\
& \text { then } H^{i}\left(P, f^{*}\left(K_{M} \otimes L^{k} \otimes A\right) \otimes \mathscr{I}_{Y}(-a E)\right)=0 \text { for } i \geq 1 .
\end{aligned}
$$

To this end, let $V=\bigoplus_{i=1}^{e} B_{i}^{*}$, so that $Y \subset P$ is the zero locus of a vector bundle map $\varepsilon: V \rightarrow \mathscr{O}_{P}$. In view of the Koszul resolution of $\mathscr{J}_{Y}$ determined by $\varepsilon$, for (1.7) it is in turn enough to prove.

$$
\begin{aligned}
& H^{i}\left(P, f^{*}\left(K_{M} \otimes L^{k} \otimes A\right)(-a E) \otimes \Lambda^{t} V\right)=0 \\
& \quad \text { for } a, i \geq 1, t \geq 1, \text { and } k \geq a d_{1}+d_{2}+\cdots+d_{e} .
\end{aligned}
$$

But $\Lambda^{t} V$ is a direct sum of line bundles of the form $f^{*} L^{-s} \otimes \mathscr{O}_{P}(t E)$ with $0 \leq s \leq d_{1}+\cdots+d_{t}$. Therefore (1.8) -and hence finally the theorem-is a consequence of

Claim 1.9. For any $1 \leq t \leq e$ and $a, i \geq 1$ :

$$
H^{i}\left(P, f^{*}\left(K_{M} \otimes L^{(k-s)} \otimes A\right) \otimes \mathscr{O}_{P}((t-a) E)\right)=0
$$

provided $k \geq a d_{1}+d_{2}+\cdots+d_{e}$ and $0 \leq s \leq d_{1}+\cdots+d_{t}$.

Proceeding by induction on $a$, assume first that $a=1$. Then by Lemma 1.4 ,

$$
H^{i}\left(P, f^{*}\left(K_{M} \otimes L^{(k-s)} \otimes A\right)((t-1) E)\right)=H^{i}\left(M, K_{M} \otimes L^{(k-s)} \otimes A\right) .
$$

But $k \geq s$ and hence the term on the right vanishes by Kawamata-Viehweg. Now once one knows Claim 1.9 for given $a \geq 1$, then the theorem follows for that value of $a$. So we can assume inductively that $H^{i}\left(P, f^{*}\left(K_{M} \otimes L^{\otimes k} \otimes A\right)\right.$ $\cdot(-a E))=0$ for $i \geq 1$ and $k \geq a d_{1}+d_{2}+\cdots+d_{e}$. This implies the case $a+1$ of Claim 1.9 when $t<a+1$, whereas when $t \geq a+1$ it follows as before from Lemma 1.3 and Kawamata-Viehweg vanishing. This completes the proof of the theorem.

The remainder of this section is devoted to spelling out some variants of Theorem 1.2 and its proof. As we stated above, there is a particularly quick argument to handle the special case $d_{1}=d_{2}=\cdots=d_{m}=d$.

Remark 1.10. Keeping the notation of Theorem 1.2, assume that $X \subset M$ is cut out by sections of $H^{0}\left(M, L^{d}\right)$. As before let $f: P \rightarrow M$ be the blowing-up of $M$ along $X$, and let $E \subset P$ be the exceptional divisor. Since $X$ is cut out by sections of $L^{d}, f^{*} L^{d}(-E)$ is globally generated, and hence nef. Therefore $f^{*}\left(K_{M} \otimes L^{k} \otimes A\right) \otimes \mathscr{O}_{P}(-a E)=K_{P} \otimes\left(f^{*} L^{d}(-E)\right)^{e+a-1} \otimes f^{*}\left(L^{k-d(e+a-1)} \otimes A\right)$, 
which is of the form $K_{P} \otimes$ (big and nef) provided that $k \geq d(e+a-1)$. Hence, by Kawamata-Viehweg vanishing,

$$
H^{i}\left(M, \mathscr{F}_{X}^{A} \otimes K_{M} \otimes L^{k} \otimes A\right)=H^{i}\left(P, f^{*}\left(K_{M} \otimes L^{k} \otimes A\right) \otimes \mathscr{O}_{P}(-a E)\right)=0
$$

for $a, i \geq 1$ and $k \geq d(e+a-1)$, as required.

Note that Severi's theorem does not follow directly from the statement of Theorem 1.2 because he does not assume as we do that $X \subset \mathbb{P}^{r}$ is defined by hypersurfaces of degrees $\leq \max \left\{d_{1}, \ldots, d_{r-2}\right\}$. However the proof of Theorem 1.2 does yield the following generalization of Severi's result, whose verification we leave to the reader.

Proposition 1.11. With notation as in the proof of Theorem 1.2, assume that $X \subset M$ lies in the complete intersection of $e=\operatorname{codim}(X, M)$ sections $s_{i} \in$ $H^{0}\left(M, L^{d_{i}}\right)$, and let $F_{i} \in\left|f^{*} L^{d_{i}}(-E)\right|$ be the corresponding divisors residual to $E$ in the blow-up $P$ of $M$ along $X$. Assume that $Y=F_{1} \cap F_{2} \cap \cdots \cap F_{e}$ is smooth, and that no component of $Y$ lies in the exceptional divisor $E$. Then $H^{i}\left(M, \mathscr{I}_{X} \otimes K_{M} \otimes L^{k} \otimes A\right)=0$ for $i \geq 1$ and $k \geq d_{1}+d_{2}+\cdots+d_{e}$.

Observe that we are not assuming here anything extra about the equations defining $X$ in $M$. The argument for Theorem 1.2 does not directly yield the analogous statement for higher power of the ideal sheaf, because if $a>1$ then the proof of Claim 1.6 requires that one know that $f^{*} L^{d_{1}}(-E)$ is generated by its global sections. It might seem more natural to assume the smoothness of the variety $X^{\prime} \subset M$ residual to $X$ in the complete intersection of the $s_{i}$-as Severi did-but when $n \geq 4, X^{\prime}$ will typically be singular.

Finally, we remark that an argument similar to the proof of Theorem 1.2 leads to an extension of a well-known vanishing theorem of Griffiths [Griff]. As at the beginning of this section, $M$ is a complex projective manifold, $L$ is a globally generated line bundle on $M$, and $A$ is a big and nef line bundle on $M$.

Proposition 1.12. Suppose that $E$ is a vector bundle of rank $e$ on $M$, which is a quotient of a direct sum of powers of $L$

$$
L^{d_{1}} \oplus \cdots \oplus L^{d_{m}} \rightarrow E \rightarrow 0,
$$

where $0 \leq d_{1} \leq \cdots \leq d_{m}$. Then

$$
H^{i}\left(M, S^{t}(E) \otimes L^{-k} \otimes \operatorname{det} E \otimes A \otimes K_{M}\right)=0
$$

for $i \geq 1, t \geq 0$, and $k \leq(t+1) d_{1}+\cdots+d_{e}$.

When $d_{1}=\cdots=d_{m}=0$, this is the Griffiths vanishing theorem for globally generated vector bundles.

Sketch of Proof of Proposition 1.12. Let $f: P=\mathbb{P} E \rightarrow M$ be the projectivization of $E$ and set $B_{i}=f^{*} L^{-d_{i}} \otimes \mathscr{O}_{\mathbb{P E}}(1)$. As in the proof of Theorem 1.2 one argues first that if $D_{i} \in\left|B_{i}\right|$ is a sufficiently general divisor, then $Y=D_{1} \cap D_{2} \cap \cdots \cap D_{e}$ 
is smooth and generically finite over $M$. Set $C_{t, k}=f^{*}\left(L^{-k} \otimes \operatorname{det} E \otimes A \otimes K_{M}\right)$ $\otimes \mathscr{O}_{\mathbb{P E}}(t)$. Applying Kawamata-Viehweg vanishing on $Y$, one finds that $H^{i}\left(Y, C_{t, k} \mid Y\right)=0$ for $i \geq 1$ and $k \leq(t+1) d_{1}+\cdots+d_{m}$. The Koszul resolution for $\mathscr{I}_{Y}$ shows that $H^{i}\left(P, C_{t, k} \otimes \mathscr{F}_{Y}\right)=0$ for $i$ and $k$ in the same range, and the assertion follows.

\section{APPLICATIONS TO SUBVARIETIES OF PROJECTIVE SPACE}

In this section we give the applications to subvarieties of projective space, as stated in the introduction.

Proof of Proposition 1. Apply Theorem 1.2 with $A=L=\mathscr{O}_{\mathbb{P}^{r}}(1)$.

Proof of Corollary 2. If $d_{1}+\cdots+d_{e} \leq r+1$, then $H^{1}\left(X, \mathscr{I}_{X}(k)\right)=0$ for $k \geq 1$ and hence $X$ is projectively normal. If $d_{1}+\cdots+d_{e} \leq r$, then in addition $H^{i+1}\left(\mathbb{P}^{r}, \mathscr{J}_{X}(k)\right)=H^{i}\left(X, \mathscr{O}_{X}(k)\right)=0$ for $k \geq 0$ and $i \geq 1$. Since $H^{i}\left(X, \mathscr{O}_{X}(k)\right)=0$ when $k<0$ and $1 \leq i \leq n-1$ by Kodaira, it follows that $X$ is projectively Cohen-Macaulay.

Proof of Corollary 5. When $X$ is a local complete intersection, Esnault [E] proves that the desired inequality is a consequence of the vanishing $H^{i}\left(\mathbb{P}^{r}, \mathcal{F}_{X}^{a}(k)\right)=0$ for $i \geq 0$ and $0 \geq k \geq a d_{1}+d_{2}+\cdots+d_{e}-r$. When $i=0$ this is clear since $k \leq 0$, and when $i \geq 1$ it is the assertion of Proposition 1.

Proof of Corollary 4. Given $X \subset \mathbb{P}^{r}$ of dimension $n$, to check that $X$ is $k$ regular (for $k \geq 0$ ) it is sufficient to verify that $H^{i}\left(\mathbb{P}^{r}, \mathscr{J}_{X}(k-i)\right)=0$ for $1 \leq i \leq n+1$, the remaining vanishings being automatic. Therefore statement (i) of the corollary is a consequence of Proposition 1.

Now assume that $X$ fails to to be $\left(d_{1}+\cdots+d_{e}-e\right)$-regular. Then it likewise follows from Proposition 1 that

$H^{n+1}\left(\mathbb{P}^{r}, \mathscr{J}_{X}\left(d_{1}+\cdots+d_{e}-e-n-1\right)\right)=H^{n}\left(X, \mathscr{O}_{X}\left(d_{1}+\cdots+d_{e}-r-1\right)\right) \neq 0$.

Equivalently,

$$
H^{0}\left(X, K_{X} \otimes \mathscr{O}_{X}\left(r+1-d_{1}-\cdots-d_{e}\right)\right) \neq 0 .
$$

Arguing as in the proof of Theorem 1.2, we may choose polynomials $s_{i} \in$ $H^{0}\left(\mathbb{P}^{r}, \mathscr{J}_{X}\left(d_{i}\right)\right) \quad(1 \leq i \leq e)$ defining hypersurfaces $D_{i} \supset X$ such that the $s_{i}$ generate $\mathscr{I}_{X}$ away from a codimension 1 subset. Thus

$$
Y={ }_{\text {def }} D_{1} \cap D_{2} \cap \cdots \cap D_{e}=X \cup X^{\prime},
$$

where $X^{\prime}$ (if nonempty) is either disjoint from $X$ or meets $X$ in a divisor. We suppose henceforth that $n=r-e \geq 1$. Then $Y$ is connected, so to show that $X=Y$, it is enough to prove that $X \cap X^{\prime}=\varnothing$. 
To this end, observe that the differentials of the $s_{i}$ determine in the natural way a map

$$
u: \mathscr{O}_{X}\left(-d_{1}\right) \oplus \cdots \oplus \mathscr{O}_{X}\left(-d_{e}\right) \rightarrow N^{*}
$$

where $N^{*}$ is the conormal bundle to $X$ in $\mathbb{P}^{r}$. Since the $s_{i}$ generically generated $\mathscr{I}_{X}, u$ is injective considered as a homomorphism of coherent sheaves. Furthermore, coker $u$ is supported on $X \cap X^{\prime}$. Computing first Chern classes, it follows that $X \cap X^{\prime}$ is supported on a divisor in the linear series

$$
\left|\operatorname{det} N^{*} \otimes \mathscr{O}_{X}\left(d_{1}+\cdots+d_{e}\right)\right|=\left|\mathscr{O}_{X}\left(d_{1}+\cdots+d_{e}-r-1\right) \otimes K_{X}^{*}\right| .
$$

In view of $(*)$, this implies that the linear series in question is trivial, and hence that $u$ is an isomorphism. Therefore $X \cap X^{\prime}=\varnothing$, and we are done.

Remark. The assertion of the corollary remains true if $\operatorname{dim} X=0$. We leave it to the interested reader to make the necessary changes to the argument just given.

Proposition 1 also leads to a quick proof of a slight generalization of a theorem of Mumford [BM] bounding the regularity of $X$ in terms of its degree.

Corollary 2.1. Assume that $X \subset \mathbb{P}^{r}$ is a smooth variety of degree $d$, dimension $n$, and codimension $e=r-n$. Set $c=\min \{e, n+1\}$. Then

$$
H^{i}\left(\mathbb{P}^{r}, \mathscr{I}_{X}(k)\right)=0 \quad \text { for } i \geq 1 \text { and } k \geq c(d-1)-n .
$$

Remark. Mumford's result is the statement that $H^{i}\left(\mathbb{P}^{r}, \mathscr{J}_{X}(k)\right)=0$ for $i \geq 1$ and $k \geq(n+1)(d-1)-n=(n+1)(d-2)+1$. When $X$ is nondegenerate, one hopes that in fact the stated vanishing holds as soon as $k \geq d+n-r$, at least when $r \geq 2 n+1$, but this is only known when $\operatorname{dim} X \leq 3$ [GLP, P, L1, R2].

Proof of Corollary 2.1. When $r \geq 2 n+1$, it is enough to prove the stated vanishing for the embedding $X \subset \mathbb{P}^{2 n+1}$ obtained by taking a general projection to $\mathbb{P}^{2 n+1}$. Therefore we may assume that $r=n+c$. But recall that if $X$ has degree $d$, then $X$ is scheme-theoretically cut out in $\mathbb{P}^{r}$ by hypersurfaces of degree $d$ (cf. [M]). Hence the corollary follows from Proposition 1.

Remark. Observe that the proof requires only the easy case $d_{1}=\cdots=d_{e}=d$ of Proposition 1.

We conclude this section by proving the "Babylonian-tower"-type ${ }^{1}$ Corollary 3 and a generalization. As before $X \subset \mathbb{P}^{r}$ denotes a smooth irreducible projective variety of dimension $n$, degree $d$, and codimension $e=r-n$. We assume that $X \subset \mathbb{P}^{r}$ is nondegenerate, i.e., that it is not contained in any hyperplanes. The first point is an elementary estimate for the number of generators of the homogeneous ideal of a Cohen-Macaulay variety.

\footnotetext{
${ }^{1}$ Results of this sort can be seen as asserting that $X \subset \mathbb{P}^{r}$ is a complete intersection provided that it is the hyperplane section of a smooth variety $X_{1} \subset \mathbb{P}^{r+1}$, where $X_{1}$ is in turn the hyperplane section of a smooth variety $X_{2} \subset \mathbb{P}^{r+2}$, and so on indefinitely-a situation that is supposed to evoke the image of a Babylonian tower.
} 
Lemma 2.2. Assume that $X \subset \mathbb{P}^{r}$ as above is projectively Cohen-Macaulay. Then the homogeneous ideal $I=I_{X}$ of $X$ can be generated by $e(d-1)-\left(\begin{array}{l}e \\ 2\end{array}\right)$ generators.

Proof. Since the homogeneous coordinate ring of $X$ is Cohen-Macaulay, we can mod out by a regular sequence without changing the number of generators of $I$. Therefore the lemma reduces to the following assertion:

Let $V$ be a vector space of dimension $e$; and let $I \subset S=$ $\operatorname{Sym}(V)$ be a homogeneous ideal of codimension $d$, generated by elements of degrees $\geq 2$, so that the graded ring $R=S / I$ is Artinian of length $d$. Let $\mathbb{C}=S / S_{+}$denote the residue field of $S$ at the irrelevant maximal ideal. Then $\operatorname{dim}_{\underline{\mathbb{C}}} \operatorname{Tor}_{1}^{S}(R, \underline{\mathbb{C}}) \leq$ $e(d-1)-\left(\begin{array}{l}e \\ 2\end{array}\right)$.

But this follows easily from the fact (cf. [G]) that one can compute the Tor in question as the homology at the middle term of the Koszul-type complex:

$$
\Lambda^{2} V \otimes R \rightarrow V \otimes R \rightarrow R .
$$

Indeed, the map on the right has rank $\geq e$, and choosing an element of $R$ gives an embedding $\Lambda^{2} V \subset \operatorname{ker}\{V \otimes R \rightarrow R\}$.

Remark. In the situation of the lemma, let $R$ be the homogeneous coordinate ring of $X$. A similar argument shows that the $k$ th module of syzygies of $I_{X}$ has $\leq\left(\begin{array}{l}e \\ k\end{array}\right)(d-1)-\left(\begin{array}{c}e \\ k+1\end{array}\right)$ minimal generators. Moreover this is sharp if $X$ is a variety of minimal degree.

Corollary 2.4. Keeping notation as above, assume that $d \leq r / 2 e$. Then $X$ is a complete intersection. If $n \geq(3 r-2) / 4$, then $X$ is a complete intersection provided that $d \leq r / e$.

Proof. Recall again that if $X \subset \mathbb{P}^{r}$ has degree $d$, then $X$ is scheme-theoretically defined by hypersurfaces of degree $d$ (cf. [M]). Therefore $X$ is projectively Cohen-Macaulay once $d e \leq r$ thanks to Corollary 2. In this case, Lemma 2.2 implies that $X$ is cut out by $\leq e d$ equations. But according to a result of Faltings [F, Satz 3], if $X \subset \mathbb{P}^{r}$ is cut out scheme-theoretically by $k \leq r / 2$ equations, then $X$ is a complete intersection. The first assertion of the corollary follows. For the second, we invoke an extension of Faltings's theorem by Netsvetaev [N], who shows that it is enough to assume $k \leq n$ provided that $n \geq(3 r-2) / 4$.

Remark. In order to underscore the fundamentally elementary nature of Corollary 3, we wish to emphasize that the quoted theorem of Faltings is quite quick to prove. Observe also that we have again only used the case $d_{1}=\cdots=d_{e}=d$ of Proposition 1. Note also that we have not used the full statement of Lemma 2.2. Doing so leads to a slightly better inequality in Corollary 2.4 , which we 
leave to the reader to formulate. We remark that Faltings's theorem is used in a similar context in Flenner's paper [F12].

\section{NORMAL GENERATION OF ADJOINT BUNDLES}

This section is devoted to Proposition 6 of the introduction and some variants. Throughout, $X$ is a smooth projective variety of dimension $n$ and $A$ is a very ample line bundle on $X$. Fix nef line bundles $B$ and $C$ on $X$, and for any integers $k, m$, put

$$
M_{k}=K_{X} \otimes A^{k} \otimes B \quad \text { and } \quad N_{m}=K_{X} \otimes A^{m} \otimes C .
$$

We denote by $p_{1}, p_{2}: X \times X \rightarrow X$ the two projections.

Lemma 3.1. The diagonal $\Delta \subset X \times X$ is cut out scheme-theoretically by sections of $L=\left(p_{1}\right)^{*} A \otimes\left(p_{2}\right)^{*} A$.

Proof. The embedding $X \subset \mathbb{P}^{r}$ defined by $A$ gives rise to an inclusion $X \times$ $X \subset \mathbb{P}^{r} \times \mathbb{P}^{r}$, and $\Delta_{X}=\Delta_{\mathbb{P}^{r}} \cap(X \times X)$. Therefore it is enough to prove that $\Delta_{\mathbb{P}^{r}} \subset \mathbb{P}^{r} \times \mathbb{P}^{r}$ is defined by sections of $\mathscr{O}_{\mathbb{P} \times \mathbb{P}}(1,1)$. But this is elementary.

Proof of Proposition 6. We use the time-honored device of reducing the question to a vanishing on $X \times X$. Specifically, consider on $X \times X$ the exact sequence

$$
0 \rightarrow\left(p_{1}\right)^{*} M_{k} \otimes\left(p_{2}\right)^{*} N_{m} \otimes \mathscr{I}_{\Delta} \rightarrow\left(p_{1}\right)^{*} M_{k} \otimes\left(p_{2}\right)^{*} N_{m} \rightarrow M_{k} \otimes N_{m} \mid \Delta \rightarrow 0 .
$$

The multiplication map $H^{0}\left(X, M_{k}\right) \otimes H^{0}\left(X, N_{m}\right) \rightarrow H^{0}\left(X, M_{k} \otimes N_{m}\right)$ is just the homomorphism on global sections induced by the restriction to $\Delta$. Consequently it is enough to prove that $H^{1}\left(X \times X,\left(p_{1}\right)^{*} M_{k} \otimes\left(p_{2}\right)^{*} N_{m} \otimes \mathscr{I}_{\Delta}\right)=0$ when $k, m \geq n+1$. But this follows from Theorem 1.2 applied to the embedding $\Delta \subset X \times X$ with $L=\left(p_{1}\right)^{*} A \otimes\left(p_{2}\right)^{*} A$. In fact,

$$
\left(p_{1}\right)^{*} M_{k} \otimes\left(p_{2}\right)^{*} N_{m}=K_{X \times X} \otimes L^{n} \otimes D,
$$

where $D=\left(p_{1}\right)^{*}\left(A^{k-n} \otimes B\right) \otimes\left(p_{2}\right)^{*}\left(A^{m-n} \otimes C\right)$. Now $L$ is certainly base-point free, and $D$ is ample provided that $k, m \geq n+1$. Therefore the required vanishing follows from Theorem 1.2 and Lemma 3.1

Variant 3.2. With assumptions as above, the multiplication map

$$
H^{0}\left(X, M_{k}\right) \otimes H^{0}\left(X, N_{m}\right) \rightarrow H^{0}\left(X, M_{k} \otimes N_{m}\right)
$$

is surjective as soon as $k, m \geq n$ unless $(X, A)=\left(\mathbb{P}^{n}, \mathscr{O}_{\mathbb{P}^{n}}(1)\right)$. In this case, surjectivity fails if either $B$ or $C$ is trivial.

Sketch of proof. Rather than trying to put this in a general setting, it is easiest in the case at hand to simply give a modification of the argument of Remark 1.10 above. Consider the blowing-up $f: P=\mathrm{Bl}_{\Delta}(X \times X) \rightarrow X \times X$ of $X \times X$ 
along the diagonal. Let $E \subset P$ denote the exceptional divisor, and set $H=$ $f^{*}\left(\left(p_{1}\right)^{*} A \otimes\left(p_{2}\right)^{*} A\right)(-E)$. Much as in Remark 1.10 the required vanishing of $H^{1}\left(P, f^{*}\left(\left(p_{1}\right)^{*} M_{k} \otimes\left(p_{2}\right)^{*} N_{m}\right)(-E)\right)$ for $k, m \geq n$ will follow from KawamataViehweg as soon as we prove

Claim 3.3. With the stated exception, $H$ is big and nef.

In fact consider the embedding $X \subset \mathbb{P}^{r}$ defined by $A$. Then there is a natural map $\lambda_{0}: X \times X-\Delta \rightarrow G=\operatorname{Grass}\left(\mathbb{P}^{1}, \mathbb{P}^{r}\right)$ that takes a pair of points to the line they span. $\lambda_{0}$ extends to a morphism $\lambda: P \rightarrow G$, and one checks (for instance as in Lemma 3.1) that $H=\lambda^{*} \mathscr{O}_{G}(1)$, where $\mathscr{O}_{G}(1)$ is the positive generator of $\operatorname{Pic}(G)$. To prove Claim 3.3 it is then enough to show that $\lambda$ is generically finite. But this is just the classical fact that unless $X=\mathbb{P}^{r}$ (which case we are excluding), a general secant line to $X$ is not contained in $X$.

Remark. By adapting an argument from [G, II], one can use these ideas to prove weak results for defining equations and higher syzygies. Specifically, one finds that if $A$ is very ample and $k \geq(p+1) n+1$, then $K_{X} \otimes A^{k}$ satisfies condition $\left(N_{p}\right)$ in the sense of [GLP] or [G]. However, stronger results are established using vector bundle methods in [EL], so we do not pursue this approach here.

Finally, we give an application to Gaussian maps. Given line bundles $N$ and $M$ on $X$, set $R(M, N)=\operatorname{ker}\left\{H^{0}(X, M) \otimes H^{0}(X, N) \rightarrow H^{0}(X, M \otimes N)\right\}$. Then one can define a map

$$
\gamma_{M, N}: R(M, N) \rightarrow H^{0}\left(X, M \otimes N \otimes \Omega_{X}^{1}\right)
$$

by making sense of

$$
s \otimes t \mapsto s \otimes d t-t \otimes d s .
$$

These homomorphisms have attracted a certain amount of attention lately, mainly on curves, starting with the work of Wahl [W1] (cf. also [W2, W3]). In particular, there is some interest in understanding when $\gamma_{M, N}$ is surjective. For curves the best-possible result is known when $N$ and $M$ have large degree [BEL]. In general one has:

Corollary 3.4. With $M_{k}$ and $N_{m}$ defined as at the beginning of this section, let $\gamma_{k, m}: R\left(M_{k}, N_{m}\right) \rightarrow H^{0}\left(X, M_{k} \otimes N_{m} \otimes \Omega_{X}^{1}\right)$ denote the corresponding Gaussian homomorphism. Then $\gamma_{k, m}$ is surjective provided that $k, m \geq n+2$.

Proof. It is enough to prove that $H^{1}\left(X \times X,\left(p_{1}\right)^{*} M_{k} \otimes\left(p_{2}\right)^{*} N_{m} \otimes \mathscr{J}_{\Delta}^{2}\right)=0$ when $k, m \geq n+2$ (cf. [W3]). But this follows as above from the case $a=2$ of Theorem 2.1.

Remark. As in [W3] one can define higher order maps involving higher powers of $\mathscr{I}_{\Delta}$. Needless to say, the same argument gives a surjectivity statement in this context as well. 


\section{REFERENCES}

[ABS] M. Andreatta, E. L. Ballico, and A. Sommese, On the projective normality of the adjunction bundles. II, preprint.

[AS] M. Andreatta and A. Sommese, On the projective normality of the adjunction bundles, preprint.

[B] W. Barth, Submanifolds of low codimension in projection space, Proc. Internat. Congr. Math., Vancouver 1974, pp. 409-413.

[BM] D. Bayer and D. Mumford (to appear).

[BS1] D. Bayer and M. Stillman, On the complexity of computing syzygies, Computational Aspects of Commutative Algebra (L. Robbianno, ed.), Academic Press, 1989, pp. 1-13.

[BS2] _ A criterion for detecting m-regularity, Invent. Math. 87 (1987), 1-11.

[BEL] A. Bertram, L. Ein, and R. Lazarsfeld, Surjectivity of Gaussian maps for line bundles of large degree on curves (to appear).

[Btl] D. Butler, Normal generation of vector bundles on curves (to appear).

[DD] P. Deligne and A. Dimca, Filtrations de Hodge et par l'ordre du pôle pour les hypersurfaces singulaires, preprint.

[EL] L. Ein and R. Lazarsfeld, $A$ theorem on the syzygies of smooth projective varieties of arbitrary dimension (to appear).

[E] E. Esnault, Hodge type of subvarieties of $\mathbb{P}^{N}$ of small degrees, preprint.

[EV1] H. Esnault and E. Viehweg, Sur une minoration du degré d'hypersurfaces s'annulant en certains points, Math. Ann. 263 (1983), 75-86.

[EV2] _ Dyson's lemma for polynomials in several variables (and the theorem of Roth), Invent. Math. 78 (1984), 445-490.

[F] G. Faltings, Ein Kriterium fur vollständig Durschnitte, Invent. Math. 62 (1981), 393-401.

[F11] H. Flenner, Babylonian tower theorems on the punctured spectrum, Math. Ann. 271 (1985), 153-160.

[F12] - Babylonian tower theorems for coverings (to appear).

[G] M. Green, Koszul cohomology and the geometry of projective varieties. I, II, J. Differential Geom. 19 (1984), 125-171, 20 (1984), 279-289.

[Griff] P. Griffiths, Hermitian differential geometry, Chern classes, and positive vector bundles, Global Analysis: Papers in honor of K. Kodaira, Princeton Univ. Press, Princeton, NJ, 1969, pp. 181-251.

[GLP] L. Gruson, R. Lazarsfeld, and C. Peskine, On a theorem of Castelnuovo and the equations defining projective varieties, Invent. Math. 72 (1983), 491-506.

[H] R. Hartshorne, Varieties of small codimension in projective space, Bull. Amer. Math. Soc. 80 (1974), 1017-1032.

[HS] A. Holme and M. Schneider, A computer aided approach to codimension 2 subvarieties of $\mathbb{P}^{n}, n \geq 6$, Crelle's J. 357 (1985), 205-220.

[K] Y. Kawamata, A generalization of Kodaira-Ramanujam's vanishing theorem, Math. Ann 261 (1982), 43-46.

[Kmf] G. Kempf, Projective coordinate rings of abelian varieties, Algebraic Analysis, Geometry and Number Theory (J. I. Igusa, ed.), Johns Hopkins Press, 1989, pp. 225-236.

[L1] R. Lazarsfeld, A sharp Castelnuovo bound for smooth surfaces, Duke Math. J. 55 (1987), 423-238.

[L2] _ _ A sampling of vector bundle techniques in the study of linear series, Lectures on Riemann Surfaces (M. Cornalba et al., eds.), World Scientific Press, 1989, pp. 500-559.

[Mori] S. Mori, Classification of higher dimensional varieties, Proc. Sympos. Pure Math., vol. 46, Amer. Math. Soc., Providence, RI, 1987, pp. 269-331. 
[M] D. Mumford, Varieties defined by quadratic equations, Corso CIME 1969, Questions on Algebraic Varieties, Rome, 1970, pp. 30-100.

[N] Y. Netsvetaev, Projective varieties defined by small numbers of equations are complete intersections (Topology and Geometry-Rohlin Seminair XI), Lecture Notes in Math., vol. 1346, Springer, 1988, pp. 433-453.

[P] H. Pinkham, A Castelnuovo bound for smooth surfaces, Invent. Math. 83 (1986), 321-332.

[R1] Z. Ran, On projective varieties of codimension 2, Invent. Math. 73 (1983), 333-336.

[R2] Local differential geometry and generic projections of threefolds, J. Differential Geom. 32 (1990), 131-137.

[Sat] E. Sato, Babylonian tower theorem on varieties, preprint.

[SR] J. Semple and L. Roth, Introduction to algebraic geometry, Oxford Univ. Press, 1949.

[S] F. Severi, Si alcune questioni di postualzione, Rend. Circ. Mat. Palermo 17 (1903), 73-103.

[V.] E. Viehweg, Vanishing theorems, J. Reine Angew. Math. 335 (1982), 1-8.

[W1] J. Wahl, The Jacobian algebra of a graded Gorenstein singularity, Duke Math J. 55 (1987), 843-871.

[W2] _ Guassian maps on algebraic curves, J. Differential Geom. 32 (1990), 77-98.

[W3] _ Gaussian maps and tensor products of irreducible representations (to appear).

Department of Mathematics, Harvard University, Cambridge, Massachusetts 02138

Department of Mathematics, University of Illinois, Chicago Circle, Chicago, Illinois 60680

Department of Mathematics, University of California, Los ANGeles, California 90024 